\title{
Deep Brain Stimulation for Movement Disorders
}

National Institute of Neurological Disorders and Stroke (NINDS)

\section{Source}

National Institute of Neurological Disorders and Stroke (NINDS). Deep Brain Stimulation for Movement Disorders Information Page.

Deep brain stimulation (DBS) is a surgical procedure used to treat several disabling neurological symptoms-most commonly the debilitating motor symptoms of Parkinson's disease (PD), such as tremor, rigidity, stiffness, slowed movement, and walking problems. The procedure is also used to treat essential tremor, dystonia, and focal epilepsy (epilepsy that originates in just one part of the brain). At present, the procedure is used only for individuals whose symptoms cannot be adequately controlled with medications. However, only individuals who improve to some degree after taking medication for Parkinson's benefit from DBS.

DBS uses a surgically implanted, battery-operated medical device called an implantable pulse generator (IPG) —similar to a heart pacemaker and approximately the size of a stopwatch to-deliver electrical stimulation to specific areas in the brain that control movement, thus blocking the abnormal nerve signals that cause symptoms. The DBS system consists of three components: the lead, the extension, and the IPG. The lead (also called an electrode) - a thin, insulated wire-is inserted through a small opening in the skull and implanted in the brain. The tip of the electrode is positioned within the specific brain area. The extension is an insulated wire that is passed under the skin of the head, neck, and shoulder, connecting the lead to the implantable pulse generator. The IPG (the "battery pack") is the third component and is usually implanted under the skin near the collarbone. In some cases it may be implanted lower in the chest or under the skin over the abdomen.

Before the procedure, a neurosurgeon uses magnetic resonance imaging or computed tomography scanning to identify and locate the exact target within the brain for surgical intervention. Generally, these areas are the thalamus, subthalamic nucleus, and globus 
pallidus.

Once the system is in place, electrical impulses are sent from the IPG up along the extension wire and the lead and into the brain. These impulses block abnormal electrical signals and alleviate motor symptoms. 